\title{
RECOVERING CORRECTIONS IN THE ANALYSIS OF INTERMITTENT DATA *
}

\author{
Beata Ziaja \\ Department of Theoretical Physics \\ Institute of Nuclear Physics \\ Radzikowskiego 152, 31-342 Cracow, Poland
}

March 1999

\begin{abstract}
The analysis of intermittent data is improved. The standard method of recovering the history of a particle cascade is proved in general not to reproduce the structure of the true cascade. The recovering corrections to the standard method are proposed and tested in the framework of multiplicative cascading models.
\end{abstract}

\section{Introduction}

The first data on possible intermittent behaviour in multiparticle production [] came from the analysis of the single event of high multiplicity recorded by the JACEE collaboration [2]. Data from some accelerator experiments [3] confirmed afterwards that there are large dynamical fluctuations appearing in the high energy multiparticle final states which manifest a scaling behaviour. Many different models have been proposed since to explain the effect 四. Some of them suggested that an underlying final state multiparticle cascade may be responsible for the scaling of multiparticle moments [4]. In this approach the intermittent data represent the last stage of the cascade, and the main problem lies in the extraction of the information on previous cascading stages which is in some way encoded in the last stage data. It should be stressed that the problem of recovering the history of the cascade may

*e-mail: beataz@qcd.ifj.edu.pl 
not be solvable if adressed generally. However, the self-similar processes which are assumed to underly the final state structure obey some scaling law. This makes many features of local dynamics dissapear, and one may expect to extract from the last stage data at least a part of information on the real cascade parameters .

The method of recovering the history of the cascade from the last stage data was proposed and applied originally to the JACEE event data. Since that time it became a standard tool of multiparticle data analysis [4], especially in the event-by-event analysis [5].

In this paper we would like to improve the standard method of analizing the intermittent data, taking into account corrections due to the recovering the history of the particle cascade in the framework of multiplicative random cascading models [6, [7. This problem has been already adressed and analized in part in Ref. [8]. Our discussion will proceed as follows. In section 2 we characterize briefly the standard method of estimation of intermittency exponents, and introduce the definition of recovering corrections. In section 3 the definition of multiplicative models is presented, and the special cases of multiplicative models: $\alpha-, p-,(p+\alpha)$-models are summarized. In section 4 the recursive equation for recovering corrections in a multiparticle model with possible neighbour-node memory is derived. Section 5 is devoted to the implementation of recovering corrections into the analysis of data. The implementation algorithm is proposed and numerically tested. Finally, in section 6 we present our conclusions.

\section{Standard estimation of intermittency exponents and recovering corrections.}

Consider a sample of $M$ bins describing an individual (intermittent) event. For simplicity assume that $M=2^{n}$, where $n$ is a natural number. We thus have $2^{n}$ numbers describing the content of each bin :

$$
x_{i}^{(n)}, \quad i=0,1, \ldots, 2^{n}-1
$$

which represent e. g. the distribution of particle density into bins. One assumes that the bin ensemble has been generated in some cascading process, and the unnormalized density moments $z_{q}^{(n)}$ for this process :

$$
z_{q}^{(n)}=\frac{1}{2^{n}} \sum_{i=0}^{2^{n}-1}\left(x_{i}^{(n)}\right)^{q}
$$

manifest a scaling behaviour parametrized by intermittency exponents $\phi_{q}$ :

$$
z_{q}^{(n)} \sim 2^{n \cdot \phi_{q}}
$$


The standard method of estimation of intermittency exponents was introduced firstly for the analysis of JACEE event [2]. The method recovered the history of the cascade in the following manner: it established the value of density moments for each cascade step, and made the linear $\chi^{2}$-fit to the points $\left(k, \log z_{q}^{(k)}\right)(k=1, \ldots, n$, $\left.\log x \equiv \log _{2} x\right)$ :

$$
\log z_{q}^{(k)}=k \cdot \phi_{q}^{\prime}+b
$$

In this way the eventual long-range correlations could be separated and would not contribute to the estimated slope $\phi_{q}$. For the assumed bin-into-two-bins splitting scheme the true value of the $x_{i}^{(n-k)}$ bin content was replaced by $y_{i}^{(n-k)}$ :

$$
y_{i}^{(n-k)}=\frac{1}{2^{k}} \sum_{j=0}^{2^{k}-1} x_{2^{k} \times i+j}^{(n)} .
$$

The intermittency exponents were extracted from the reconstructed moments $z_{q ; \text { rec. }}^{(k)}$ :

$$
z_{q ; \text { rec. }}^{(k)}=\frac{1}{2^{k}} \sum_{j=0}^{2^{k}-1}\left(y_{j}^{(k)}\right)^{q},
$$

assuming their power law behaviour :

$$
z_{q ; \text { rec. }}^{(k)} \sim 2^{k \cdot \phi_{q}^{\prime}} .
$$

So far (see e. g. [10]) one has estimated the value of (normalized) $\phi_{q}$, assuming simply that $\phi_{q ; \text { norm. }}=\phi_{q}^{\prime}\left(\phi_{q ; \text { norm. }}:=\phi_{q}-q \phi_{1}\right)$.

There is, however, an open question if and how the cascade recovered from data refers to the true cascade which generated the data. For the purpose of estimating the intermittency exponents it is enough to ask about the relation between the true density moments $z_{q}$ and the reconstructed ones obtained from (6). It is obvious that formula (5) looses a piece of information on the primary cascade. We give a simple example to illustrate the problem. If we assume that the underlying cascading process preserves e. g. the total particle density $\sum_{j=0}^{2^{k}-1} x_{j}^{(k)}=1(k=1, \ldots, n)$, then it follows from (6) that the reconstructed cascade will not manifest this property: $\sum_{j=0}^{2^{k}-1} y_{j}^{(k)} \neq 1$. It means that standard method does not recover the conservation law present in the true cascade.

Moreover, it was found explicitly for the $\alpha$-model [6, 7] (which does not preserve the total particle density) that there exists always discrepancy between the true value of $\phi_{q}$ and its estimation $\phi_{q}^{\prime}(7)$, due to recovering technique (5). This problem was discussed in detail in Ref. [11].

Assume we may express the discrepancy between the true and the reconstructed moments at the $(n-k)$ th cascade step in a following way :

$$
z_{q ; r e c .}^{(n-k)}=z_{q}^{(n-k)} \cdot p_{q}(k),
$$


where the factor $p_{q}(k)$ denotes the corrections due to recovering procedure (5). We will call them recovering corrections. Corrections $p_{q}(k)$ contain information on the parameters of a specific process which generated the true cascade. It is obvious that they depend also on the cascade step. Substituting (8) into (田) one arrives at the relation :

$$
\log z_{q ; \text { rec. }}^{(n-k)}-\log \left(p_{q}(k)\right)=(n-k) \cdot \phi_{q ; \text { corr. }}+b,
$$

where the fitted slope $\phi_{q ; \text { corr }}$. estimates the true intermittency exponent $\phi_{q}$.

In this paper we confine ourselves to recovering corrections considered for the class of multiplicative random cascading models. For the multiplicative cascade ("multiplicative" means that at each cascade step the bin content is multiplied by a number to generate the bin content at the next cascade step) relation (8) holds explicitly, and recovering corrections take the form [11] (for proof see Appendix A) :

$$
p_{q}(k)=\left\langle\left(\frac{1}{2^{k}} \sum_{i=0}^{2^{k}-1} x_{i}^{(k)}\right)^{q}\right\rangle
$$

where the average $\langle\ldots\rangle$ is taken over the (eventual) random choices while generating the cascade. The starting bin $x_{0}^{(0)}$ is set equal 1 . It is worth noticing that $p_{q}(k)$ may be also expressed in terms of the erraticity moments [9] :

$$
p_{q}(k)=C_{1, q}
$$

\section{Multiplicative models}

As already mentioned, in our paper we restrict ourselves to the class of multiplicative random cascading processes with possible neighbour-node memory, generating the uniform distribution of particle density. The commonly used models of random cascading: $\alpha$-model [12 and $p$-model [13 belong to this class. For the purpose of testing our predictions for recovering corrections we introduce below a new many parameter $(p+\alpha)$-model.

In the multiplicative random cascading processes with possible neighbour-node memory assume for simplicity the root of a cascade to be equal to $1: x_{0}^{(0)}=1$. One generates the next stages of the cascade recursively. The scheme is following. The two bins $x_{2 i}^{(k+1)}$ and $x_{2 i+1}^{(k+1)}$ are obtained from $x_{i}^{(k)}$ by multiplication :

$$
\begin{aligned}
& x_{2 i}^{(k+1)}:=W_{1} \cdot x_{i}^{(k)}, \\
& x_{2 i+1}^{(k+1)}:=W_{2} \cdot x_{i}^{(k)},
\end{aligned}
$$

where $W_{1}$ and $W_{2}$ are random variables of the $m$ model parameters $a_{j}, j=1, \ldots, m$ : 


$$
\begin{aligned}
& W_{1}=a_{j} \text { with probability } p_{a_{j}} \\
& W_{2}=a_{j} \text { with probability } p_{a_{j}}
\end{aligned}
$$

with normalized probability weights $p_{a_{j}}$ :

$$
\sum_{j=1}^{m} p_{a_{j}}=1 .
$$

The distribution of particle density will be uniform if the following condition is fulfilled :

$$
p\left(W_{1}=a_{i}, W_{2}=a_{j}\right)=p\left(W_{1}=a_{j}, W_{2}=a_{i}\right),
$$

where $p\left(W_{1}=a_{i}, W_{2}=a_{j}\right)$ denotes probability of choosing in (12) $W_{1}=a_{i}$ and $W_{2}=a_{j}(i, j=1, \ldots, m)$. Then the density moments fulfill relation (3), where intermittency exponents $\phi_{q}$ are equal to :

$$
\phi_{q}=\log \left(a_{1}^{q} p_{a_{1}}+\ldots+a_{m}^{q} p_{a_{m}}\right) .
$$

The models : $\alpha-, p-$ and $(p+\alpha)$ may be derived from general multiplicative rule (12). To obtain the $\alpha$-model it is enough to assume random variables $W_{1}, W_{2}$ to be independent :

$$
\left\langle W_{1} W_{2}\right\rangle=\left\langle W_{1}\right\rangle\left\langle W_{2}\right\rangle
$$

The $\alpha$-model has no node memory therefore no conservation law can be implemented here.

Relation (12) reduces to the $p$-model after turning $m=2$ and :

$$
\begin{aligned}
a_{2}= & 1-a_{1}, \\
p_{a_{1}}= & p_{a_{2}}=0.5, \\
p\left(W_{2}=a_{2}\right. & \left.W_{1}=a_{1}\right)=1, \\
p\left(W_{2}=a_{1}\right. & \left.W_{1}=a_{2}\right)=1,
\end{aligned}
$$

where $p\left(W_{2}=a_{i} \mid W_{1}=a_{j}\right)$ denotes conditional probability of $W_{2}=a_{i}$, if $W_{1}=a_{j}$. The $p$-model is an example of a multiplicative model with the neighbour-node memory. Relation (18) implies that the sum $x_{2 i}^{(k+1)}+x_{2 i+1}^{(k+1)}=x_{i}^{(k)}$, and the particle density in a node is preserved.

Finally we introduce the many parameter $(p+\alpha)$-model, using relation (12) combined with the $\alpha$ - and $p$-model restrictions :

$$
\begin{aligned}
a_{2 i} & =1-a_{2 i-1}, \\
p_{a_{2 i}} & =p_{a_{2 i-1}},
\end{aligned}
$$


where $m$ is an even number $\left(i=1, \ldots, \frac{m}{2}\right)$, and :

$$
\begin{array}{c|l}
p\left(W_{2}=a_{2 i}\right. & \left.W_{1}=a_{2 i-1}\right)=1 \\
p\left(W_{2}=a_{2 i-1}\right. & \left.W_{1}=a_{2 i}\right)=1 .
\end{array}
$$

One may check that the particle distribution generated in the $(p+\alpha)$-model is uniform. The $(p+\alpha)$-model may involve any number of parameters $a_{i}$. Therefore it describes a more realistic case of cascading since for large $m$ the distributions of particle density for $W_{1}$ and $W_{2}$ (13) may be approximated by a continuous distribution function $f(x): W_{1,2}=x$ with probability $f(x) d x$. The total particle density will be preserved for any $m$, according to (20).

\section{Recovering corrections in multiplicative models}

Now we calculate explicitly the correction $p_{q}(k)$ for any multiplicative model with possible neighbour-node memory. To do this we will split the bins $\left(x_{i}\right.$ 's) appearing in (10) into a left half $\left(i<2^{k-1}\right)$ and a right half $\left(i \geq 2^{k-1}\right)$ [11:

$$
\begin{aligned}
p_{q}(k) & =\left\langle\left(\frac{1}{2^{k}} \sum_{i} l_{i}+r_{i}\right)^{q}\right\rangle= \\
& =\frac{1}{2^{q}}\left\langle\sum_{j=0}^{q}\left(\begin{array}{c}
q \\
j
\end{array}\right)\left(\frac{1}{2^{k-1}} \sum_{i} l_{i}\right)^{j}\left(\frac{1}{2^{k-1}} \sum_{i} r_{i}\right)^{q-j} W_{1}^{j} W_{2}^{q-j}\right\rangle .
\end{aligned}
$$

Using the fact that the left and right bins are independent, one arrives at the recurrence equation:

$$
p_{q}(k)=\frac{1}{2^{q}} \sum_{j=0}^{q}\left(\begin{array}{c}
q \\
j
\end{array}\right) p_{j}(k-1) p_{q-j}(k-1)\left\langle W_{1}^{j} W_{2}^{q-j}\right\rangle
$$

which may be solved recursively together with the initial data :

$$
\begin{aligned}
& p_{q}(0)=1 \\
& p_{0}(k)=1
\end{aligned}
$$

A similar recurrence relation has been also obtained in [8]. It should be stressed that coefficients $\left\langle W_{1}^{j} W_{2}^{q-j}\right\rangle$ are the only parameters of the model needed to solve (22) recursively. It means that to calculate $p_{q}(k)$ for a given model we need only to know the coefficients $\left\langle W_{1}^{j} W_{2}^{q-j}\right\rangle$. In the next section we show how to apply this observation to the data analysis. 
Finally we present recovering corrections calculated for the $\alpha-$ and $p$-models :

$$
\begin{aligned}
p_{q}^{\alpha-\text { model }}(k) & =\frac{1}{2^{q}} \sum_{j=0}^{q}\left(\begin{array}{c}
q \\
j
\end{array}\right) p_{j}(k-1) p_{q-j}(k-1) 2^{\phi_{j}+\phi_{q-j}}, \\
p_{q}^{p-\text { model }}(k) & =2^{\phi_{1} q k}
\end{aligned}
$$

where $\phi_{j}$ denote intermittency exponent (16).

\section{Implementation of the recovering corrections}

The idea how to implement the corrections $p_{q}(k)$ into the analysis of the $\alpha$-model data was sketched briefly in [1]. Here we extend the primary scheme, and apply it to the multiplicative model data. As it was mentioned in the previous section, coefficients $\left\langle W_{1}^{j} W_{2}^{q-j}\right\rangle$ are the only parameters of the model needed to calculate recursively the corrections $p_{q}(k)$. Let us introduce a new notation for $\left\langle W_{1}^{j} W_{2}^{l}\right\rangle$ :

$$
\left\langle W_{1}^{j} W_{2}^{l}\right\rangle \equiv k_{j, l}
$$

We ask now how to derive $k_{j, q-j}$ from the model. One may notice that for either $j=0$ or $l=0$ coefficients $k_{j, l}$ equal :

$$
k_{j, 0}=k_{0, j}=2^{\phi_{j}},
$$

where $\phi_{j}$ 's are ordinary intermittency exponents (3) which may be determined from relations (4), (9). To find the value of $k_{j, l}(j, l \neq 0)$ we use the unnormalized density correlators $c_{j, l}^{(k)}$ 迎, 幽, 14:

$$
c_{j, l}^{(k)}=\frac{1}{2^{k-1}} \sum_{i=0}^{2^{k-1}-1}\left(x_{2 i}^{(k)}\right)^{j}\left(x_{2 i+1}^{(k)}\right)^{l} .
$$

In multiplicative models the correlators and the density moments fulfill the relation :

$$
c_{j, l}^{(k)}=z_{j+l}^{(k-1)} \cdot k_{j, l}
$$

which can be also rewritten as :

$$
\log c_{j, l}^{(k)}=(k-1) \phi_{j+l}+\log k_{j, l} .
$$

Relation (29) may be easily derived from (12) : since each term in sum (28) originates

from one node $x_{i}^{(k-1)}$, it can be rewritten as : $\left(x_{2 i}^{(k)}\right)^{j}\left(x_{2 i+1}^{(k)}\right)^{l}=\left(x_{i}^{(k-1)}\right)^{j+l} W_{1}^{j} W_{2}^{l}$. Relations (29), (30) imply that we may derive $k_{j, l}$ in a straigthforward way by 
calculating correlators and density moments from data, and applying to them the standard $\chi^{2}-$ fit.

Applying the standard method to the correlators at the previous cascade stages, we expect to find the discrepancy (due to the recovering procedure) between reconstructed correlators and the true ones, similarly as for the density moments. It can be proved (see Appendix B) that the discrepancy may be expressed in terms of recovering correction $p_{q}(k)(10)$ :

$$
c_{j, l ; r e c .}^{(n-k)}=c_{j, l}^{(n-k)} p_{j+l}(k) .
$$

Now we have all tools needed for implementation of recovering corrections into the multiplicative data analysis. Below we propose an implementation algorithm which recursively adjusts the primary parameters $\phi_{q}, k_{j, l}(j+l=q, j l>0)$ obtained after applying the standard method to the data :

(INPUT) parameters $\boldsymbol{\phi}_{1}, \ldots, \boldsymbol{\phi}_{q-1}, \boldsymbol{k}_{j, l}(j+l=1, \ldots, q-1)$ obtained after applying the implementation algorithm for $q=1,2, \ldots, q-1$ step-by-step (for determination of $\phi_{1}$ see Appendix C.3),

(1) derive $\boldsymbol{\phi}_{q}^{\prime}, \boldsymbol{k}_{j, q-j}^{\prime}(j=1, \ldots, q-1)$ from data, using the standard method i. e. reconstruct the cascade using (5) and derive the parameters from relations :

$$
\begin{gathered}
\log z_{q ; \text { rec. }}^{(k)}=k \cdot \phi_{q}^{\prime}+b . \\
c_{j, l ; \text { rec. }}^{(k)}=z_{j+l ; \text { rec. }}^{(k-1)} \cdot k_{j, l}^{\prime}
\end{gathered}
$$

where $k=1, \ldots, n$ (cf. (四), (29)),

(2) derive $\phi_{q ; \text { corr. }}, \boldsymbol{k}_{j, q-j ; \text { corr. }}(j=1, \ldots, q-1)$ in the following substeps :

(2.0) calculate $p_{q}(k)$ from relation (cf. (22)) :

$$
p_{q}(k)=\frac{1}{2^{q}} \sum_{j=0}^{q}\left(\begin{array}{c}
q \\
j
\end{array}\right) p_{j}(k-1) p_{q-j}(k-1) k_{j, q-j},
$$

using $\boldsymbol{\phi}_{q}^{\prime}, \boldsymbol{k}_{j, q-j}^{\prime}$ derived in step (1), and estimate $\boldsymbol{\phi}_{q ; \text { corr. }}$ from (cf. (9)) :

$$
\log z_{q ; r e c .}^{(n-k)}-\log \left(p_{q}(k)\right)=(n-k) \cdot \phi_{q ; \text { corr. }}+b,
$$


(2.1) calculate $p_{q}(k)$ from (B4) using $\phi_{q ; \text { corr }}$ (other parameters as after step (1)),

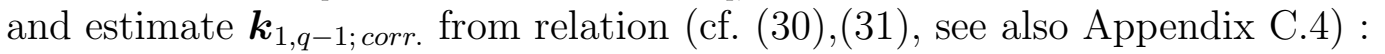

$$
\log c_{j, l ; r e c .}^{(n-k)}-\log \left(p_{j+l}(k)\right)=(n-k-1) \phi_{j+l}+\log k_{j, l ; c o r r .},
$$

$\cdots$,

(2.q-1) calculate $p_{q}(k)$ from (34), using all previously derived parameters $\boldsymbol{\phi}_{q ; \text { corr. }}$, $\boldsymbol{k}_{j, q-j ; c o r r .}$, and estimate $\boldsymbol{k}_{q-1,1 ; c o r r}$. from (36),

(3) compare the values of $\boldsymbol{\phi}_{q}^{\prime}, \boldsymbol{k}_{j, q-j}^{\prime}$ and $\boldsymbol{\phi}_{q ; \text { corr. }}, \boldsymbol{k}_{j, q-j ; \text { corr. }}(j=1, \ldots, q-1)$. If the relative difference is large, assume :

$$
\begin{aligned}
\phi_{q}^{\prime} & :=\phi_{q ; \text { corr } .} \\
k_{j, q-j}^{\prime} & :=k_{j, q-j ; c o r r} .
\end{aligned}
$$

and repeat steps $(2),(3)$ recursively until the relative difference between parameters before and after step (2) is small enough. Then go to the output, assuming $\phi_{q}:=\phi_{q}^{\prime}$, $\boldsymbol{k}_{j, q-j}:=\boldsymbol{k}_{j, q-j}^{\prime}$

(OUTPUT) parameters $\boldsymbol{\phi}_{1}, \ldots, \boldsymbol{\phi}_{q} ; \boldsymbol{k}_{j, l}(j+l=1, \ldots, q)$.

Other techniqual details and problems which may appear when applying the algorithm to data are listed in Appendix C.

We have performed numerical simulations of the $\alpha-, p-$ and $(p+\alpha)$-models in order to test how the implementation algorithm works in practice. We generated 10000 cascades of the 10 step length for the $\alpha$ - and $(p+\alpha)$-models, and one cascade of the 10 step length for the $p$-model 1 for two different parameter sets separately.

Implementation algorithm analized the data of the last cascade step. For each event it estimated the value of normalized intermittency exponents $\phi_{2 ; \text { norm. }}, \phi_{3 ; \text { norm. }}$. $\left(\phi_{i ; \text { norm. }}:=\phi_{i}-i \cdot \phi_{1}\right)$, using the standard method (step 1$)$ with recovering corrections included (steps 2,3). The results are presented in Figs. 1, 2, 3, 4 (for the $\alpha$ - and $(p+\alpha)$-models $)$ and in Tabs. 1, 2 .

For the $\alpha$-model the histograms of $\phi_{2 ; n o r m .}, \phi_{3 ; \text { norm. }}$ obtained in the standard method and the histograms with recovering corrections included are almost identical. In this case the recovering corrections can be implemented better when one applies directly dedicated $\alpha$-model recovering correction $p_{q}^{\alpha-\text { model }}(k)$ (24) (see Figs. 1, 2 and Tab. 1). The different accuracy of these both approaches is due to the fact that

\footnotetext{
${ }^{1}$ it can be proved that for a given parameter set the $p$-model generates always the same values of the correlators and density moments
} 
the random variables $W_{1}, W_{2}$ are independent in the $\alpha$-model, and the coefficients $k_{j, q-j}(26)$ are approximated better by the product $2^{\phi_{j}} \cdot 2^{\phi_{q-j}}$ than from correlators (29).

On the contrary, the implementation algorithm works well for the $(p+\alpha)$-model (see Figs. 3, 4 and Tab. 2). For the $(p+\alpha)$-model the histogram with the recovering corrections included approximates well the theoretical value of normalized intermittency exponent. The histogram obtained by using the standard method is moved slightly to the left in comparison to the histogram with recovering corrections included.

We have checked that for the $p$-model the theoretical values of normalized intermittency exponents are estimated perfectly by both standard method and implementation algorithm, as we have expected 4 .

It should be also mentioned that the histograms generated by both implementation algorithm and dedicated recovering corrections are symmetric, in contrast to the standard ones, and their dispersions are relatively small (see Tabs. 1, 2). Finally, we notice that the accuracy of the estimation of intermittency exponents, while applying the standard method and the improved one to a given model, depends on the parameters of this model (cf. Figs. 1, 2 and Figs. 3, 4). In Appendix D we present a qualitative analysis of the effect for intermittency exponents of the second rank. A similar analysis has also been done in [8].

\section{Conclusions}

To sum up we analized the estimation of intermittency exponents from the data which were generated by a multiplicative random cascading process. The following methods were applied: the standard method of cascade recovering (5) and the improved method which included recursively the recovering corrections. The improved method was applied in the form of the implementation algorithm. Numerical simulations have been performed to check how both methods work in practice. The conclusions may be summarized as follows :

(a) standard method of estimation of intermittency exponents does not apply for the whole class of multiplicative models : its accuracy depends on the specific properties of the model and its parameters. The method does not detect a conservation law if present in the model;

(b) improved method of estimation of intermittency exponents applied in the form of recursive implementation algorithm either corrects the standard method

\footnotetext{
${ }^{2}$ it follows from relations (8),(25) that in the $p$-model $\phi_{i ; \text { rec. }, \text { norm. }}=\phi_{i ; n o r m . ~}$ if $i>1$
} 
estimation or does not change the standard method result. In the latter case the estimation may be corrected by applying the dedicated recovering corrections. In any case the improved method tests the applicability of the standard method, and allows one to estimate the accuracy of the intermittency exponents estimation.

While applying the improved method, the parameters of the model are adjusted recursively from the primary (standard method) parameters. The histograms generated by the improved method are symmetric, and their dispersions are of the same order as those determined for the standard method. The improved method takes into account the neighbour-node memory (a conservation law) if present in the model, by including the density correlators into the estimation scheme.

\section{Acknowledgements}

I would like to thank Prof. A. Białas for reading the manuscript and many suggestions and comments and Dr. R. Janik for discussions. This work was supported in part by Polish Government grant Project (KBN) 2P03B04214.

\section{Appendix A}

We prove relation $(10)$. The density moment $z_{q ; \text { rec. }}^{(n-k)}$ may be rewritten as :

$$
z_{q ; \text { rec. }}^{(n-k)}=\frac{1}{2^{n-k}} \sum_{i=0}^{2^{n-k}-1}\left(y_{i}^{(n-k)}\right)^{q}=\frac{1}{2^{n-k}} \sum_{i=0}^{2^{n-k}-1}\left(\frac{1}{2^{k}} \sum_{j=0}^{2^{k}-1} x_{2^{k} \times i+j}^{(n)}\right)^{q} .
$$

Notice that :

$$
x_{2^{k} \times i+j}^{(n)}=x_{i}^{(n-k)} \cdot x_{j}^{(k)} .
$$

Substituting (38) into (37), one arrives at :

$$
z_{q ; \text { rec. }}^{(n-k)}=\frac{1}{2^{n-k}} \sum_{i=0}^{2^{n-k}-1}\left(x_{i}^{(n-k)}\right)^{q}\left(\frac{1}{2^{k}} \sum_{j=0}^{2^{k}-1} x_{j}^{(k)}\right)^{q}=z_{q}^{(n-k)} p_{q}(k) .
$$

\section{Appendix B}

We prove relation (31). The correlator $c_{j, l ; \text { rec. }}^{(n-k)}$ may be rewritten as :

$$
c_{j, l ; \text { rec. }}^{(n-k)}=\frac{1}{2^{n-k-1}} \sum_{i=0}^{2^{n-k-1}-1}\left(y_{2 i}^{(n-k)}\right)^{j}\left(y_{2 i+1}^{(n-k)}\right)^{l}
$$




$$
=\frac{1}{2^{n-k-1}} \sum_{i=0}^{2^{n-k-1}-1}\left(\frac{1}{2^{k}} \sum_{m=0}^{2^{k}-1} x_{2^{k} \times 2 i+m}^{(n)}\right)^{j}\left(\frac{1}{2^{k}} \sum_{r=0}^{2^{k}-1} x_{2^{k} \times(2 i+1)+r}^{(n)}\right)^{l} .
$$

Relation (38) implies :

$$
c_{q ; \text { rec. }}^{(n-k)}=\frac{1}{2^{n-k-1}} \sum_{i=0}^{2^{n-k-1}-1}\left(x_{2 i}^{(n-k)}\right)^{j}\left(x_{2 i+1}^{(n-k)}\right)^{l}\left(\frac{1}{2^{k}} \sum_{j=0}^{2^{k}-1} x_{j}^{(k)}\right)^{j+l}=c_{j, l}^{(n-k)} p_{j+l}(k) .
$$

\section{Appendix C}

We list some techniqual details which can be useful when applying the implementation algorithm to data, and discuss possible problems.

(1) The recovering corrections applied for calculating $\phi_{2}, \phi_{3}$ read :

$$
\begin{aligned}
p_{1}(k) & =2^{k \phi_{1}} \\
p_{2}(k) & =\frac{1}{4}\left(p_{2}(k-1) k_{2,0}+p_{2}(k-1) k_{0,2}+2 p_{1}^{2}(k-1) k_{1,1}\right) \\
p_{3}(k) & =\frac{1}{8}\left(p_{3}(k-1) k_{3,0}+p_{3}(k-1) k_{0,3}\right. \\
& \left.+3 p_{1}(k-1) p_{2}(k-1) k_{1,2}+3 p_{2}(k-1) p_{1}(k-1) k_{2,1}\right)
\end{aligned}
$$

(2) Since random variables $W_{1}, W_{2}$ generate the uniform distribution (cf. (15)) relations (42,43,44) may be simplified by substituting :

$$
k_{j, l}=k_{l, j} .
$$

The (experimental) estimation of $k_{j, l}$ will be better if we determine $c_{j, l}^{(n)}$ as :

$$
c_{j, l}^{(n)}=\frac{1}{2^{n-1}} \sum_{i=0}^{2^{n-1}-1}\left\{\left(x_{2 i}^{(n)}\right)^{j}\left(x_{2 i+1}^{(n)}\right)^{l}+\left(x_{2 i}^{(n)}\right)^{l}\left(x_{2 i+1}^{(n)}\right)^{j}\right\} .
$$

(3) Determination of $\phi_{1}$. It follows from relations (8), (42) that the unnormalized

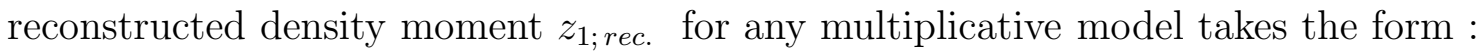

$$
z_{1 ; r e c .}^{(k)}=2^{n \phi_{1}}=z_{1}^{(n)}
$$

which does not depend on the cascade stage $k$. Therefore the proposed estimation of $\phi_{1}$, implied by (47) reads :

$$
\phi_{1}=\frac{\log z_{1 ; r e c}^{(n)}}{n}
$$


(4) Estimation of coefficients $k_{j, l}$ in the implementation algorithm. To estimate coefficients $k_{j, l}$ in the step (1) of the implementation algorithm we calculate the reconstructed correlators and density moments, and apply relation (33) whereas in the step (2) we use relation (36) to do the same.

The brief explanation of the method is following. In formula (33) $k_{j, l}$ appears as a slope, and an ordinary linear $\chi^{2}$-fit may estimate it with a good accuracy. This approach works well for the reconstructed correlators and moments.

On the contrary, the long-range correlations: $\log b$ present in relation (36) add to the value of $\log k_{j, l}$, and generate a large error while estimating $k_{j, l}:=\exp \left(\log k_{j, l}+\right.$ $\log b$ ) from (36). We could try to estimate $b$, assuming that :

$$
z_{q}^{(n)}=2^{n \cdot \phi_{q}} b
$$

Then it follows from (36) that :

$$
\log c_{j, l}^{(n)}=(n-1) \phi_{j+l}+\log \bar{k}_{j, l},
$$

and $\log k_{j, l}$ equals :

$$
\log k_{j, l}=\log \bar{k}_{j, l}-\log b .
$$

The latter approach does not work for the reconstructed moments, where relations (49,50) apply only approximately. However, it applies quite well for the moments with recovering corrections included because for this case relation (33) would require including recovering corrections to both correlators and density moments which in turn would generate larger error in estimating $k_{j, l}$.

It was checked that the above method works for the multiplicative model data. However, the problem of determination of coefficients $k_{j, l}$ and, in particular, the

problem of the determination of correlators $c_{j, l}^{(n)}$ from the real data is much more complicated (see e. g. [四, 14]). In this case the method needs some improvement which we will not discuss here.

(5) The recursive implementation algorithm is not always convergent. Since estimation at the $q$ th step depends upon parameters which were adjusted in the previous steps $1, \ldots, q-1$, the estimation errors propagate and get larger with growing $q$. Then it happens sometimes (not very often), that recursive adjusting ends with the repeating a sequence of different values of parameters, or parameters become not definite. In such a case we stop the algorithm, assuming for the values of intermittency parameters those derived in step (1).

\section{Appendix D}

The accuracy of the estimation of intermittency exponents, while applying the standard method and the improved one to a given model, depends on the parameters of 
this model (cf. Figs. 1, 2 and Figs. 3, 4). Below we present a qualitative analysis of the effect for the intermittency exponents of the second rank.

Equation (43) may be solved analytically, and the solution (valid for any multiplicative model) takes the closed form :

$$
p_{2}(k)=(1-A) \cdot 2^{\left(\phi_{2}-1\right) k}+A \cdot 2^{2 k \phi_{1}},
$$

where :

$$
A=\frac{k_{1,1}}{2^{2 \phi_{1}+1}-2^{\phi_{2}}} .
$$

The reconstructed moments then read :

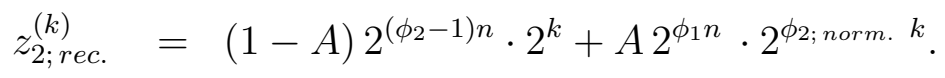

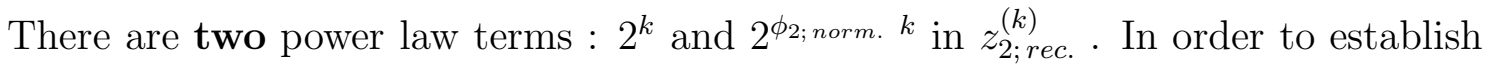
how they influence the determination of $\phi_{2}^{\prime}(1,32)$ we performed the following check. For a given multiplicative model with fixed parameters (e. g. $\alpha$ - model with param-

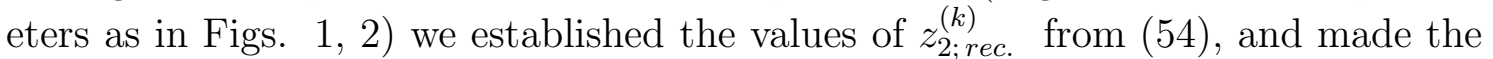
linear $\chi^{2}$-fit to the points $\left(k, \log z_{2 ; r e c .}^{(k)}\right)$. The slope obtained from the fit estimated the value of $\phi_{2}^{\prime}$. For the $\alpha$-model we have obtained : for case a) $\phi_{2}^{\prime}=0.0250$, and for case b) $\phi_{2}^{\prime}=0.284$. Both results agree with the $\phi_{2}^{\prime}$ 's obtained from the model simulation (cf. Figs. 1,2 and Tab. 1). Similar analysis can be also peformed for the $(p+\alpha)-$ model.

The above results confirm our observation that the accuracy of the estimation of intermittency exponents from the standard method depends on the parameters of the model. And, if the estimation of primary parameters $\phi_{q}^{\prime}, k_{j, l}^{\prime}$ is more accurate, their recursive adjusting performed by the implementation algorithm will be faster

and more accurate as well. It means that in this case the improved analysis works better as well.

\section{References}

[1] A. Bialas, R. Peschanski, Nucl. Phys. B273, 703 (1986); A. Bialas, R. Peschanski, Nucl. Phys. B207, 59 (1988)

[2] JACEE coll. T. H. Burnett et al. , Phys. Rev. Lett. 50 (1983) 2062,

[3] P. Carlson (UA5), 4th Topical Workshop on P-P Collider Physics, Bern, March 1983; G. J. Alner et al., Phys. Rep. 154, (1987) 247,

[4] for recent reviews see

E. A. de Wolf, I. M. Dremin, W. Kittel, Phys. Rep. 270 (1996) 1,

P. Bozek, M. Ploszajczak, R. Botet, Phys. Rep. 252 (1995) 101, 
[5] A. Bialas, R. Hwa, Phys. Lett. B253 (1991) 436; L. Lianshou, F. Jinghua, W. Yuanfang, HZPP 9807,

[6] R. Peschanski, Nucl. Phys. B327 (1989) 144; P. Brax, R. Peschanski, Nucl. Phys. B346 (1990) 65; A. Bialas, R. Peschanski, Phys. Lett. B207 (1988) 59; H. C. Eggers, M. Greiner, P. Lipa, Phys. Rev. Lett. 80 (1998) 5333; H. C. Eggers, M. Greiner, P. Lipa, hep-ph 9811204,

[7] A. Bialas, A. Szczerba, K. Zalewski, Z. Phys. C46 (1990) 163,

[8] P. Desvallees, R. Ouziel, R. -Peschanski, Phys. Lett. B235 (1990) 317; Y. Gabellini, J. -L. Meunier, R. Peschanski, Z. Phys. C55 (1992), 455,

[9] R. Hwa, Acta Phys. Pol. B27 (1996) 1789,

[10] A. Bialas, B. Ziaja, Phys. Lett. B378 (1996) 319,

[11] R. Janik, B. Ziaja, Acta Phys. Pol. B (1999) 259,

[12] D. Shertzer, S. Lovejoy, Proc. IUATM Symp. on Turbulence and Chaotic Phenomena in Fluids, Kyoto, Sept. 1983 (North-Holland, Amsterdam, 1984); D. Shertzer, S. Lovejoy, Selected Papers from the 4th Symp. on Turbulent Shear Flows, University of Karlsruhe (1983), ed. L. J. S. Bradbury et al. (Springer Verlag, 1984)

[13] C. Meneveau, K. R. Sreenivasan, Phys. Rev. Lett. 59 (1987) 1424,

[14] R. Peschanski, J. Seixas, preprint CERN-TH-5903-90, 


\section{Figure captions}

Figs. 1, 2 Estimation of normalized intermittency exponents $\phi_{2 ; \text { norm. }}$ and $\phi_{3 ; \text { norm }}$. for $\alpha$-model, using the standard method (dotted line), the improved method with the implementation algorithm (thin solid line), and dedicated $\alpha$-corrections (24) (dashed line) compared with the theoretical values (solid line), performed for two sets of $\alpha$-model parameters :

a) $a_{1}=0.8, a_{2}=1.1, p_{1}=1 / 3$

b) $a_{1}=0.5, a_{2}=1.5, p_{1}=1 / 2$.

Figs. 3, 4 Estimation of normalized intermittency exponents $\phi_{2 ; \text { norm. }}$ and $\phi_{3 ; \text { norm. }}$. for $(p+\alpha)$-model, using the standard method (dotted line), the improved method with the implementation algorithm (thin solid line), compared with the theoretical values (solid line), performed for two sets of $(p+\alpha)$-model parameters :

a) $a_{2 i}=1-a_{2 i-1}, p_{2 i}=p_{2 i-1}=0.05$ for $i=1, \ldots, 10$,

$a_{1}=0.2, a_{3}=0.5, a_{5}=0.6, a_{7}=0.3, a_{9}=0.45$,

$a_{11}=0.25, a_{13}=0.1, a_{15}=0.15, a_{17}=0.87, a_{19}=0.66$,

b) $a_{2 i}=1-a_{2 i-1}, p_{2 i}=p_{2 i-1}$ for $i=1, \ldots, 10$,

$a_{1}=0.2, a_{3}=0.5, a_{5}=0.6, a_{7}=0.3, a_{9}=0.45$,

$a_{11}=0.25, a_{13}=0.1, a_{15}=0.15, a_{17}=0.87, a_{19}=0.66$,

$2 p_{1}=0.05,2 p_{3}=0.15,2 p_{5}=0.25,2 p_{7}=0.40,2 p_{9}=0.05$,

$2 p_{11}=0.05,2 p_{13}=0.02,2 p_{15}=0.02,2 p_{17}=0.005,2 p_{19}=0.005$, 
Tab. 1 Estimation of normalized intermittency exponents $\phi_{2 ; \text { norm. }}$ and $\phi_{3 ; n o r m}$. and their dispersions for the $\alpha$-model, using the standard method (second column), the improved method with the implementation algorithm (third column), and dedicated $\alpha$ - corrections (24) (fourth column), compared with the theoretical values (first column), performed for two sets of $\alpha$-model parameters (see Figs. 1, 2) :

\begin{tabular}{|r|c|c|c|c|}
\hline \hline & theor. & standard & algorithm & $\alpha$-corr. \\
\hline a) $\phi_{2 ; \text { norm. }}$ & 0.0285 & $0.0251 \pm 0.004$ & $0.0246 \pm 0.0033$ & $0.0288 \pm 0.004$ \\
\hline$\phi_{3 ; \text { norm. }}$ & 0.0813 & $0.0757 \pm 0.010$ & $0.0727 \pm 0.009$ & $0.0798 \pm 0.0111$ \\
\hline \hline $\mathrm{b}) \phi_{2 ; \text { norm. }}$ & 0.322 & $0.264 \pm 0.044$ & $0.253 \pm 0.050$ & $0.276 \pm 0.051$ \\
\hline$\phi_{3 ; \text { norm. }}$ & 0.807 & $0.653 \pm 0.105$ & $0.666 \pm 0.125$ & $0.750 \pm 0.131$ \\
\hline \hline
\end{tabular}

Tab. 2 Estimation of normalized intermittency exponents $\phi_{2 ; n o r m}$. and $\phi_{3 ; n o r m}$. and their dispersions for the $(p+\alpha)$-model, using the standard method (second column), the improved method with the implementation algorithm (third column), compared with the theoretical values (first column), performed for two sets of $(p+\alpha)$-model parameters (see Figs. 3, 4) :

\begin{tabular}{|r|c|c|c|}
\hline \hline & theor. & standard & algorithm \\
\hline a) $\phi_{2 ; \text { norm. }}$ & 0.333 & $0.322 \pm 0.044$ & $0.305 \pm 0.066$ \\
\hline$\phi_{3 ; \text { norm. }}$ & 0.832 & $0.736 \pm 0.118$ & $0.720 \pm 0.174$ \\
\hline \hline b) $\phi_{2 ; \text { norm. }}$ & 0.177 & $0.170 \pm 0.023$ & $0.173 \pm 0.029$ \\
\hline$\phi_{3 ; \text { norm. }}$ & 0.478 & $0.438 \pm 0.069$ & $0.470 \pm 0.092$ \\
\hline \hline
\end{tabular}



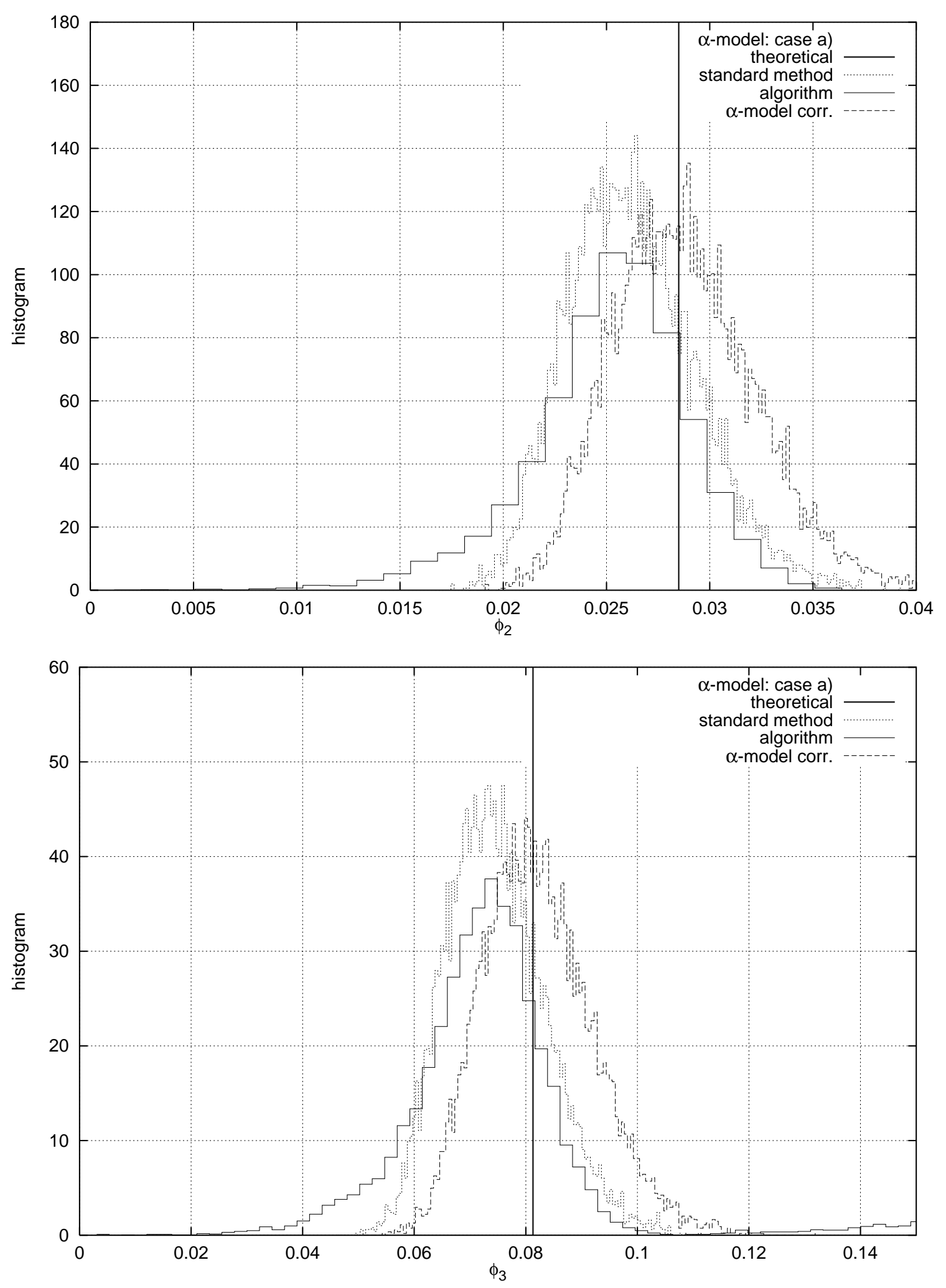

Figure 1: 

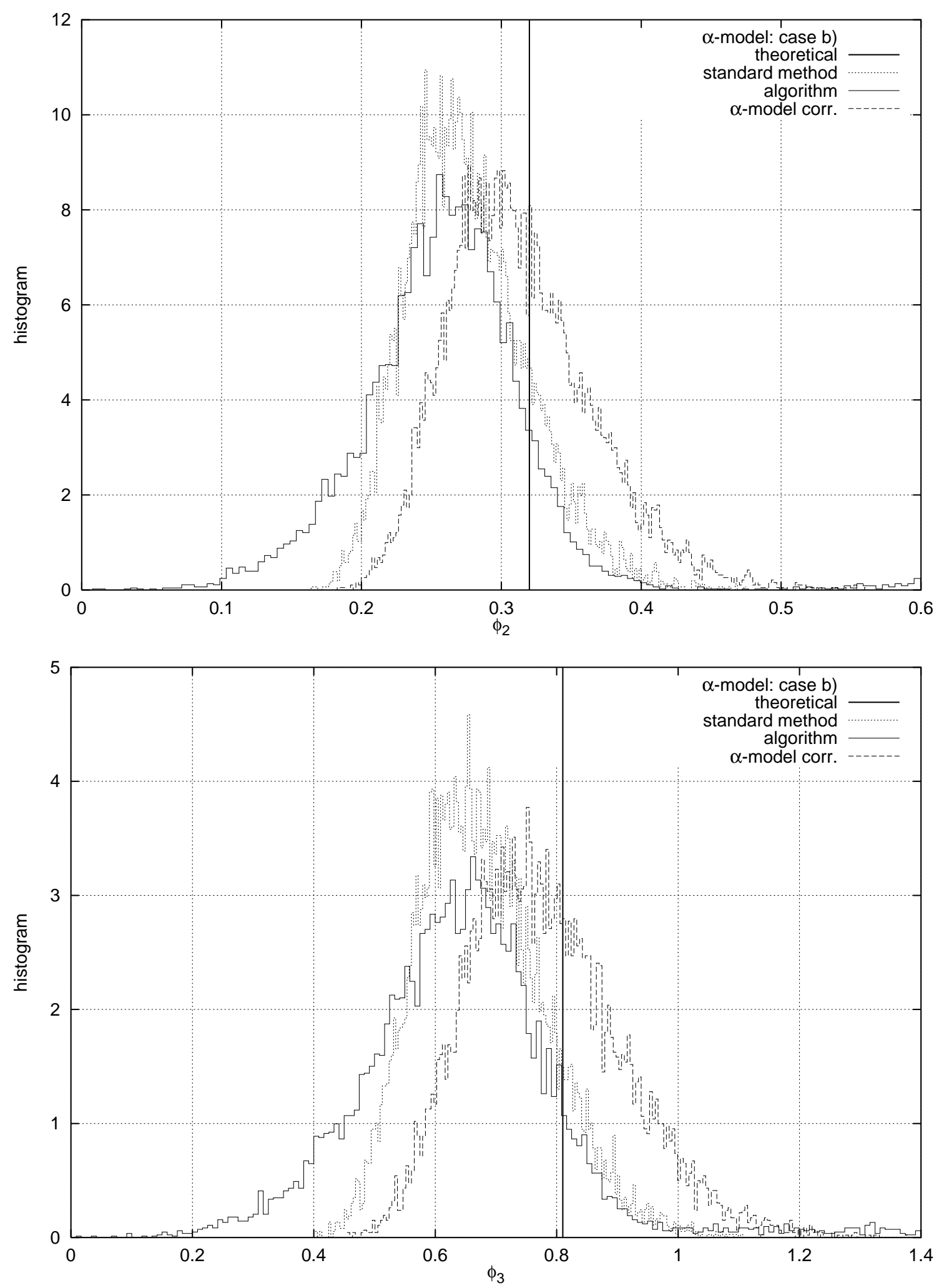

Figure 2: 

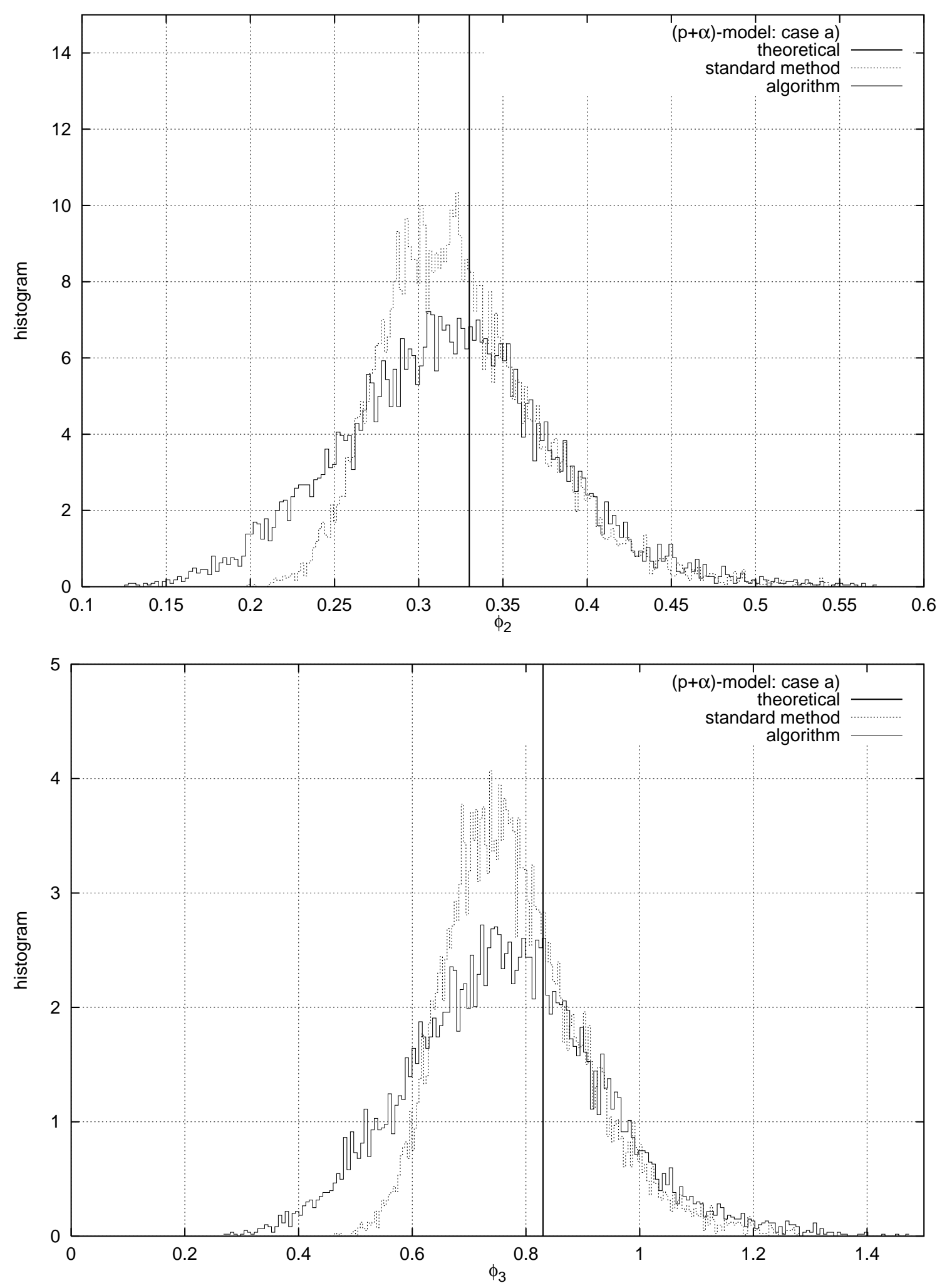

20

Figure 3: 

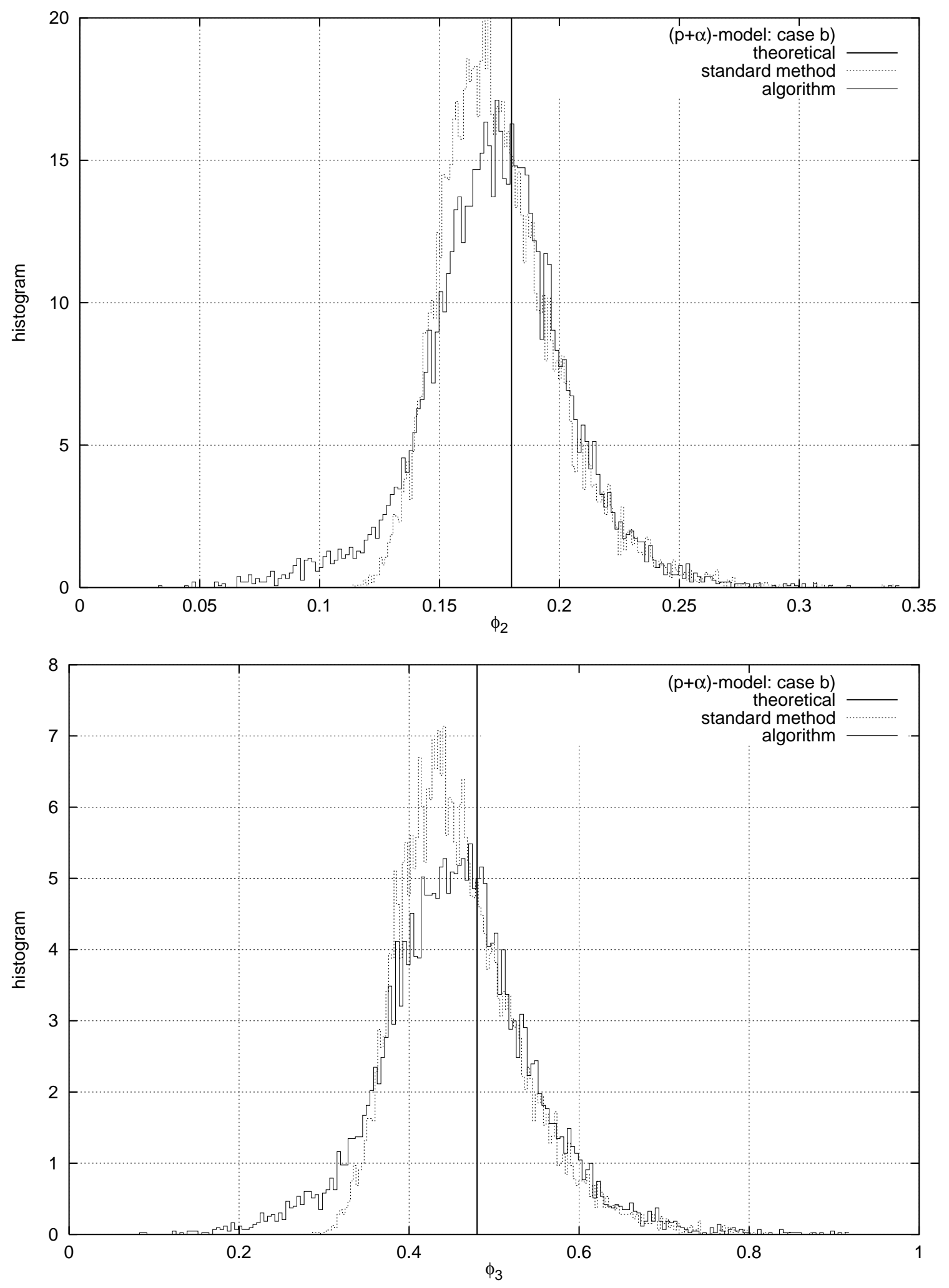

21

Figure 4: 